\title{
ESTABILIDADE DE AGREGADOS DE UM LATOSSOLO AMARELO DISTROCOESO DE TABULEIRO COSTEIRO SOB DIFERENTES APORTES DE RESÍDUOS ORGÂNICOS DA CANA-DE-AÇÚCAR ${ }^{(1)}$
}

\author{
Romero Falcão Bezerra de Vasconcelos ${ }^{(2)}$, José Ramon Barros \\ Cantalice $^{(3)}$, Veronildo Souza de Oliveira ${ }^{(4)}$, Yuri Daniel Jatobá da \\ Costa $^{(5)} \&$ Douglas Monteiro Cavalcante ${ }^{(6)}$
}

\begin{abstract}
RESUMO
O potencial produtivo da cana-de-açúcar decorre de fatores relacionados ao clima, ao solo e à variedade cultivada. Sistemas de manejo que proporcionem a adição de resíduos orgânicos ao solo podem provocar alterações em seus atributos físicos, favorecendo o crescimento do sistema radicular e a produtividade da canade-açúcar. O objetivo deste trabalho foi avaliar os efeitos de diferentes sistemas de manejo com cana-de-açúcar com diversos níveis de adição de resíduos orgânicos sobre a distribuição e os índices de estabilidade de agregados de um Latossolo Amarelo distrocoeso dos tabuleiros costeiros de Alagoas. Foram selecionadas áreas em talhões de produção com a cultura da cana-de-açúcar: uma área cultivada sob sistema de manejo irrigado (SMI), uma área sob sistema de manejo de fertirrigação com vinhaça (SMV) e uma área sob sistema de manejo com aplicação de vinhaça + torta de filtro (SMVT). Esses sistemas de manejo foram comparados entre si e em relação a uma testemunha-padrão, representada por um fragmento de Mata Atlântica (MN). Para avaliar a distribuição e os índices de estabilidade de agregados, amostras do solo foram coletadas ao acaso, nas áreas submetidas aos
\end{abstract}

\footnotetext{
(1) Parte da Tese de Doutorado do primeiro autor apresentada ao Departamento de Agronomia da Unidade Universidade Federal Rural de Pernambuco - UFRPE. Recebido para publicação em abril de 2009 e aprovado em dezembro de 2009.

(2) Professor do Departamento de Tecnologia Rural, Universidade Federal Rural de Pernambuco - UFRPE. Av. Dom. Manoel de Medeiros s/n, Dois Irmãos, CEP 52171-900 Recife (PE). E-mail: romerofalcao@hotmail.com

(3) Professor do Departamento de Agronomia, UFRPE. E-mail: cantalic@terra.com.br

(4) Professor do Departamento de Tecnologia Rural, UFRPE. E-mail: verofat@dtr.ufrpe.br.

${ }^{(5)}$ Professor do Departamento de Engenharia Civil, Universidade Federal do Rio Grande do Norte - UFRN. Campus Universitário s/n, Lagoa Nova, CEP 59072-970 Natal (RN). E-mail: ydjcosta@ct.ufrn.br

${ }^{(6)}$ Graduando em Engenharia Agronômica, UFRPE. E-mail: dougagronomo@yahoo.com.br
} 
diferentes sistemas de manejo, nas profundidades de 0-0,20, 0,20-0,40 e 0,40-0,60 m, sendo retiradas num ponto situado nas entrelinhas da cana-de-açúcar. Os resultados foram submetidos à análise de variância e as médias comparadas pelo teste de Tukey a $5 \%$. Estudos de correlações simples foram realizados entre algumas variáveis medidas. Os resultados permitiram concluir que os diferentes sistemas de manejo promoveram alterações na distribuição de agregados do solo e a estabilização dos agregados na camada superficial dependeu do teor de matéria orgânica e da ação dos ciclos sucessivos de umedecimento e secagem do solo. Os estudos de correlações entre o carbono orgânico total e os índices de estabilidade dos agregados apresentaram correlações significativas e positivas.

Termos de indexação: tamanho de agregados, manejo da cana-de-açúcar, solos coesos.

\title{
SUMMARY: AGGREGATE STABILITY IN A DYSTROPHIC COHESIVE YELLOW LATOSOL OF A COSTAL PLAIN UNDER DIFFERENT SUGARCANE RESIDUE APPLICATION
}

\begin{abstract}
The potential yield of sugarcane depends on factors related to climate, soil and the plant variety. Management systems that provide the addition of organic waste to the soil can cause changes in physical attributes of the soil, enhancing the growth of the root system and sugarcane yield. The objective of this study was to evaluate the effects of different management systems at several levels of organic residue addition on the distribution and indices of aggregate stability of dystrophic cohesive Yellow Latosol of coastal plains, in the State of Alagoas. Plots were selected in the following sugarcane-growing areas: area under irrigation management system, area fertigated with vinasse, and an area under application of vinasse + filter cake. These management systems were compared to each other and to a control (native forest). To evaluate the distribution and stability indices of soil aggregates, soil samples were randomly collected in the different areas, at the depths $0-0.20 ; 0.20-0.40$ and $0.40-0.60 \mathrm{~m}$, at points between two neighboring sugarcane rows. The results were subjected to analysis of variance and the mean compared by the Tukey test at $5 \%$ significance. Simple correlation analysis was performed between some measured variables. Results showed that the different management systems induced changes in the aggregate distribution and that the stabilization of soil aggregates in the surface layer dependeds on the organic matter content and the action of successive cycles of soil wetting and drying. The studies of simple correlations showed that total organic $C$ and aggregate stability indices were significantly and positively correlated.
\end{abstract}

Index terms: aggregate stability, sugarcane management, cohesive soils.

\section{INTRODUÇÃO}

O solo, quando submetido a cultivos intensivos, tende a perder a estrutura original, pelo fracionamento dos agregados maiores em unidades menores, havendo, como consequência, diminuição de macroporos e aumento de microporos e da densidade (Carpenedo \& Mielniczuk, 1990).

Chinn \& Pillai (2008) e Richard et al. (2001) comentam que a compactação do solo, dependente do conteúdo de água, pode causar mudanças significantes na estrutura, em termos de redução da porosidade total e da continuidade dos poros, distribuição do seu diâmetro e estabilidade deles. Essas alterações estruturais, por sua vez, levam a mudanças em três indicadores comuns da estrutura do solo: porosidade preenchida pelo ar, densidade do solo e resistência às tensões aplicadas (McNabb et al., 2001). Por isso, as estratégias de manejo do solo para melhorar ou recuperar a estrutura, como o cultivo com rotação de culturas e a incorporação de matéria orgânica (Milne $\&$ Haynes, 2004), objetivam aumentar a porosidade do solo, ao mesmo tempo em que reduzem a densidade e dissipam a energia de cargas aplicadas.

A agregação pode ser definida como a união de partículas (argila - íon - matéria orgânica, areia e silte) na unidade estrutural do solo (o agregado), sendo sua estabilidade caracterizada como a resistência a uma ação mecânica degradante, particularmente da água (Chaves \& Calegari, 2001). A agregação do solo 
controla os movimentos internos de água, ar e calor e o crescimento de raízes. Os sistemas de manejo conservacionistas aumentam os estoques de matéria orgânica e a estabilidade de agregados (Balesdent et al., 2000). Os resíduos orgânicos diminuem a densidade do solo e criam poros de diâmetro grande, que favorecem a entrada de ar e a drenagem de água.

A movimentação mecânica do solo provoca aumento da oxidação de compostos orgânicos e perdas por lixiviação e erosão. Segundo Silva et al. (2006), o cultivo com cana-de-açúcar, em comparação com a mata nativa, reduziu a estabilidade dos agregados em água e o diâmetro médio ponderado dos agregados obtidos via úmida, e as raízes das plantas e os micélios de fungos criam uma rede que favorece a estabilidade dos agregados, os quais aumentam em número na presença de gramíneas, quando o solo não é perturbado. Esses autores verificaram correlação positiva e significativa entre o diâmetro médio ponderado dos agregados (via úmida) e os teores de $\mathrm{C}$ orgânico total, demonstrando a importância dos compostos orgânicos na estabilização dos agregados do solo. A contribuição da matéria orgânica na agregação do solo é um processo dinâmico, sendo recomendável o acréscimo contínuo de material orgânico para manter a estrutura necessária ao desenvolvimento das plantas.

Wendling et al. (2005), pesquisando a influência de diferentes manejos no C orgânico e na estabilidade de agregados em Latossolo, concluíram que o plantio direto aumenta os índices de agregação em relação ao preparo convencional, mas diminui em relação à mata nativa; observaram também boa correlação do teor de C orgânico com os índices de estabilidade de agregados.

A agregação é uma das propriedades que podem ser utilizadas para avaliar a qualidade do solo, uma vez que a manutenção de sua estrutura facilita a aeração e a infiltração de água e reduz a erodibilidade (Neves et al., 2006). Esses pesquisadores verificaram que a estabilidade de agregados foi reduzida quando o solo passou da vegetação de floresta para culturas. Essa redução foi mais acentuada no solo com culturas anuais e com pomar capinado e menor naquele sob pomar com cobertura vegetal de gramíneas ou leguminosas.

A mobilização mecânica do solo para o plantio e cultivo da cana-de-açúcar tem maiores efeitos na estabilidade de agregados e na condutividade hidráulica em relação à matéria orgânica e ao tempo de cultivo no Latossolo Vermelho distroférrico (Góes et al., 2005).

Com a remoção da vegetação natural e subsequentes anos de cultivo, ocorre redução gradativa da aptidão cultural do solo, pela alteração de suas propriedades físicas. Para Wohlenberg et al. (2004), a maior estabilidade estrutural em solo arenoso ocorreu sob campo natural, e a maior desagregação, sob solo descoberto, devido ao intensivo preparo do solo, que reduziu o teor de matéria orgânica e aumentou a quantidade de agregados de menor diâmetro. Esses autores também constataram ação direta das culturas na formação e estabilização dos agregados, tendo ocorrido estabilidade maior em sistemas de manejo que adicionaram material orgânico e cobriram o solo durante o ano todo.

Diante do exposto, este trabalho objetivou avaliar os efeitos de diferentes sistemas de manejo da canade-açúcar com diversos aportes de resíduos orgânicos sobre a distribuição e os índices de estabilidade de agregados de um Latossolo Amarelo distrocoeso dos tabuleiros costeiros de Alagoas.

\section{MATERIAL E MÉTODOS}

O trabalho experimental foi conduzido na Fazenda Pau Amarelo, de propriedade da Usina Santa Clotilde S/A, compreendida entre as coordenadas $9^{\circ} 27^{\prime} 31^{\prime \prime} \mathrm{S}$, $35^{\circ} 51^{\prime} 09$ "W; 9०27' 13 " S, 35 ${ }^{\circ} 51^{\prime} 12$ "W; $9^{\circ} 27^{\prime} 13$ " S, $35^{\circ} 50$ ' 56 "W; e 9 ${ }^{\circ} 27^{\prime} 32$ " S, $35^{\circ} 50$ ' 54 " W, que têm como referência horizontal o datum WGS84, localizada no município de Rio Largo, litoral do Estado de Alagoas. O clima do local onde foi realizada a pesquisa é tropical chuvoso com verão seco, segundo a classificação de Köppen. A precipitação pluvial média anual situa-se próximo a $1.752 \mathrm{~mm}$, e a temperatura média anual, em torno de $29^{\circ} \mathrm{C}$.

As áreas escolhidas como experimentais são cultivadas com a cana-de-açúcar há mais de 20 anos, sendo o relevo de plano a suave ondulado, característico dos solos dos Tabuleiros Costeiros. O solo estudado foi classificado como Latossolo Amarelo distrocoeso (Embrapa, 2006) relevo plano textura média/argilosa, formado por sedimentos arenoargilosos do Grupo Barreiras, característico da unidade geomorfológica Tabuleiros Costeiros (Jacomine et al., 1975).

As áreas selecionadas estão sob três sistemas de manejo adotados pela unidade sucroalcooleira desde a safra 2003/2004, constituindo, dessa forma, os tratamentos avaliados: uma área cultivada sob sistema de manejo irrigado (SMI), outra área cultivada sob sistema de manejo de fertirrigação com vinhaça (SMV) e a última com a aplicação de vinhaça + torta de filtro (SMVT). Esses sistemas de manejo foram comparados entre si e em relação a uma testemunhapadrão, reproduzida por solo em condições naturais de um fragmento de Mata Atlântica (MN).

Na área cultivada sob SMI foi aplicada uma lâmina de $120 \mathrm{~mm}$ de água por ciclo da cana-de-açúcar, cuja composição química média encontra-se no quadro 1. A adubação química da cana-planta foi realizada de acordo com a análise do solo, sendo aplicados 40, 120 e $90 \mathrm{~kg} \mathrm{ha}^{-1}$ de $\mathrm{N}, \mathrm{P}_{2} \mathrm{O}_{5}$ e $\mathrm{K}_{2} \mathrm{O}$, respectivamente, e na cana-soca foram aplicados 80,50 e $90 \mathrm{~kg} \mathrm{ha}^{-1} \mathrm{de} \mathrm{N}$, $\mathrm{P}_{2} \mathrm{O}_{5}$ e $\mathrm{K}_{2} \mathrm{O}$, respectivamente.

$\mathrm{Na}$ área cultivada sob SMV foram aplicados, por ciclo da cana-de-açúcar, $300 \mathrm{~m}^{3} \mathrm{ha}^{-1}$ de vinhaça diluída (Quadro 1), utilizando sistema de aspersão. Na adubação química da cana-planta foram aplicados $40 \mathrm{~kg} \mathrm{ha}^{-1}$ de $\mathrm{N}$ e, na cana-soca, $80 \mathrm{~kg} \mathrm{ha}^{-1}$ de N. 
Quadro 1. Composição química média da água de irrigação nas áreas cultivadas, da vinhaça diluída aplicada e da torta de filtro aplicada

\begin{tabular}{|c|c|c|c|c|c|c|}
\hline \multicolumn{7}{|c|}{ Água de irrigação } \\
\hline \multirow[t]{2}{*}{$\overline{\mathrm{pH}}$} & $\mathrm{Ca}^{2+}$ & $\mathrm{Mg}^{2+}$ & $\mathrm{Na}^{+}$ & $\mathbf{K}^{+}$ & $\mathrm{Cl}^{-}$ & \multirow{3}{*}{$\begin{array}{c}\mathbf{C E} \\
\mathrm{d} \mathrm{S} \mathrm{m}^{-1} \\
0,23\end{array}$} \\
\hline & & $\mathrm{mm}$ & $\mathrm{nol}_{\mathrm{c}} \mathrm{L}^{-1}$ & & & \\
\hline \multirow[t]{2}{*}{6,9} & 0,13 & 0,21 & 1,4 & 0,16 & 1,63 & \\
\hline & \multicolumn{6}{|c|}{ Vinhaça diluída } \\
\hline$\overline{\mathrm{CE}}$ & pH & $\mathbf{N}$ & $\mathbf{P}_{2} \mathbf{O}_{5}$ & $\mathrm{~K}_{2} \mathrm{O}$ & $\mathrm{Ca}^{2+}$ & $\mathrm{Mg}^{2+}$ \\
\hline \multirow{3}{*}{$\begin{array}{c}\mathrm{dSm}^{-1} \\
3,1\end{array}$} & & & & $\mathrm{~g} \mathrm{~L}^{-1}$ & & \\
\hline & 5,9 & $8,9 \quad 0,53$ & 0,17 & 0,92 & 0,26 & 0,12 \\
\hline & \multicolumn{6}{|c|}{ Torta de filtro } \\
\hline \multirow[t]{2}{*}{$\mathbf{C}$} & $\mathrm{N}$ & $\mathrm{P}_{2} \mathrm{O}_{5}$ & $\mathrm{~K}_{2} \mathrm{O}$ & $\mathrm{Ca}^{2+}$ & $\mathrm{Mg}^{2+}$ & $\overline{\mathrm{H}_{2} \mathrm{O}}$ \\
\hline & & - $\mathrm{g} \mathrm{kg}$ & 1 & & & $\mathrm{~kg} \mathrm{~kg}^{-1}$ \\
\hline 60,4 & 3,2 & 1,4 & 2,1 & 14,6 & 3,3 & 0,71 \\
\hline
\end{tabular}

CE: condutividade elétrica.

$\mathrm{Na}$ área cultivada com SMVT foram aplicados $30 \mathrm{Mg} \mathrm{ha}^{-1}$ de torta (Quadro 1), associada com vinhaça, utilizando sistema de aspersão. Na adubação química da cana-planta foram aplicados $40 \mathrm{~kg} \mathrm{ha}^{-1} \mathrm{de} \mathrm{N} \mathrm{e,} \mathrm{na}$ cana-soca, $80 \mathrm{~kg} \mathrm{ha}^{-1}$ de $\mathrm{N}$.

Nas três áreas experimentais cultivadas com canade-açúcar foram cumpridas normas rígidas de controle de tráfego de máquinas. O manejo cultural no plantio e na renovação do canavial e a mobilização mecânica do solo são descritos a seguir, nas três áreas estudadas. O preparo periódico do solo foi feito pelo uso de subsoladores de três elementos ativos de hastes parabólicas, cuja forma de mobilização é constituída por cortes verticais com intensa vibração no interior do solo. Em seguida, usaram-se grades de discos de dupla ação, com a finalidade de destruir a socaria velha e, ao mesmo tempo, provocar o deslocamento horizontal do solo, deixando-o em condições para o plantio. Finalmente, foram abertos sulcos de aproximadamente $0,25 \mathrm{~m}$ de profundidade. O plantio foi realizado manualmente, sendo colocadas no fundo do sulco as canas-sementes, que posteriormente foram cobertas com solo. Em todas as três áreas cultivadas, a lavoura da cana-de-açúcar foi queimada antes da operação de colheita.

Para caracterização física do solo, amostras foram coletadas ao acaso, nas áreas submetidas aos diferentes sistemas de manejo, nas profundidades de $0-0,20$, $0,20-0,40$ e $0,40-0,60 \mathrm{~m}$, num ponto situado nas entrelinhas da lavoura da cana-de-açúcar. A coleta do solo foi feita no mês de fevereiro de 2007. As análises físicas e químicas do solo foram realizadas no Laboratório de Física do Solo e no Laboratório de Solo/Eecac (Estação Experimental de Cana-de-Açúcar do Carpina), ambos da Universidade Federal Rural de Pernambuco (UFRPE).

$\mathrm{Na}$ análise granulométrica utilizou-se o método do densímetro de Boyoucos e, na dispersão física do solo, empregou-se um agitador mecânico durante 15 min, sendo usado também dispersante químico (Embrapa, 1997). A densidade das partículas foi determinada pelo método do balão volumétrico e a densidade do solo, por meio de anel volumétrico (Embrapa, 1997). O teor de matéria orgânica foi estimado com base no C orgânico do solo, conforme método descrito pela Embrapa (1997). No quadro 2 são apresentadas as características granulométricas, densidades do solo e de partículas e o C orgânico total do Latossolo Amarelo distrocoeso estudado.

Quadro 2. Caracterização do Latossolo Amarelo distrocoeso, sob diferentes sistemas de manejo e profundidades estudados

\begin{tabular}{|c|c|c|c|c|c|c|c|}
\hline \multirow{2}{*}{ Profundidade } & \multicolumn{3}{|c|}{ Granulometria } & & \multicolumn{2}{|c|}{ Densidade } & \multirow{2}{*}{$\operatorname{COT}^{(1)}$} \\
\hline & Areia & Silte & Argila & & Solo & Partícula & \\
\hline $\mathrm{cm}$ & 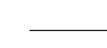 & $\mathrm{g} \mathrm{kg}^{-1}$ & - & \multirow[t]{4}{*}{ Mata (MN) } & \multicolumn{2}{|c|}{$\mathrm{Mg} \mathrm{m}^{-3}$} & $\%$ \\
\hline $0-20$ & 655 & 90 & 255 & & 1,05 & 2,62 & 3,05 \\
\hline $20-40$ & 553 & 140 & 307 & & 1,21 & 2,64 & 1,85 \\
\hline $40-60$ & 547 & 110 & 343 & & 1,27 & 2,68 & 1,23 \\
\hline $0-20$ & 680 & 70 & 250 & \multirow[t]{3}{*}{ Irrigado (SMI) } & 1,65 & 2,67 & 1,26 \\
\hline $20-40$ & 580 & 80 & 340 & & 1,72 & 2,63 & 0,87 \\
\hline $40-60$ & 572 & 98 & 330 & & 1,63 & 2,66 & 0,80 \\
\hline \multicolumn{8}{|c|}{ Vinhaça + Torta (SMVT) } \\
\hline $0-20$ & 625 & 100 & 275 & \multirow{6}{*}{ Vinhaça (SMV) } & 1,49 & 2,61 & 1,59 \\
\hline $20-40$ & 520 & 110 & 360 & & 1,56 & 2,68 & 0,85 \\
\hline $40-60$ & 437 & 120 & 443 & & 1,58 & 2,67 & 0,69 \\
\hline $0-20$ & 588 & 90 & 322 & & 1,56 & 2,64 & 1,51 \\
\hline $20-40$ & 550 & 90 & 360 & & 1,63 & 2,62 & 0,68 \\
\hline $40-60$ & 435 & 110 & 455 & & 1,62 & 2,64 & 0,60 \\
\hline
\end{tabular}

(1) COT: C orgânico total. 
A estabilidade dos agregados foi obtida por vias seca e úmida, sendo as amostras coletadas ao acaso, com o auxílio de enxadão e pá de corte. Na separação de agregados por via úmida, adotou-se o procedimento descrito por Kemper \& Rosenau (1986), com uma modificação. No tamisamento em água no aparelho de Yoder, adicionou-se a peneira de $3,35 \mathrm{~mm}$ ao conjunto de 2,00; 1,00; 0,50; e 0,25 mm de malha, o que se justifica pela necessidade de avaliação de macroagregados, que podem atingir 3,0 mm (Golchin et al., 1997; Roscoe \& Machado, 2002). Assim, $100 \mathrm{~g}$ dos agregados selecionados foram pré-umedecidos por capilaridade, transferidos para dois conjuntos com as cinco peneiras supracitadas e submetidos a agitação vertical (42 ciclos por min) durante 15 min, imersos em recipiente com água. O solo retido em cada peneira foi acondicionado em recipientes de $\mathrm{Al}$, para secagem em estufa a $105^{\circ} \mathrm{C}$, durante $24 \mathrm{~h}$. Em seguida, pesou-se e calculou-se a massa e a percentagem de agregados estáveis em cada uma das classes de diâmetro de agregados. Na separação por via seca, utilizou-se o mesmo conjunto de peneiras superpostas, mas em um agitador eletromagnético Via Test, na frequência de $90 \mathrm{rpm}$, durante $15 \mathrm{~min}$. O solo retido em cada peneira foi seco em estufa a $105^{\circ} \mathrm{C}$, durante $24 \mathrm{~h}$, sendo então realizados os mesmos cálculos supracitados. Os valores de diâmetro médio ponderado (DMP) foram obtidos de acordo com a expressão de Kemper \& Rosenau (1986):

$$
D M P=\sum_{i=1}^{n} u_{i} W_{i}
$$

em que DMP = diâmetro médio ponderado $(\mathrm{mm})$; ui = diâmetro médio entre classes $(\mathrm{mm})$; e Wi = fração em peso da classe em relação ao peso da amostra total.

Também foram obtidos o índice de estabilidade de agregados (IEA) e a percentagem de agregados maiores que 2,0 mm estáveis em água (Agri), desenvolvidos por Wendling et al. (2005), segundo as equações a seguir:

$$
\begin{array}{r}
I E A=\left(D P M_{u} / D P_{s}\right) 100 \\
A G R I=\left(W_{u}>2\right) 100
\end{array}
$$

em que $\mathrm{DMP}_{\mathrm{u}}=$ diâmetro médio ponderado via úmida $(\mathrm{mm}) ; \mathrm{DMP}_{\mathrm{s}}=$ diâmetro médio ponderado via seca (mm); e W $\mathrm{u}_{\mathrm{u}}=$ proporção de agregados $>2 \mathrm{~mm}$ estáveis em água.

Os dados obtidos foram submetidos à análise de variância e as médias comparadas pelo teste de Tukey a $5 \%$, utilizando o programa SAEG (2005). Estudos de correlação foram executados entre algumas variáveis medidas, utilizando o programa Statistica 6.0 (2001).

\section{RESULTADOS E DISCUSSÃO}

\section{Distribuição dos agregados do solo}

Inicialmente, observa-se que, para todas as condições de manejo da cana-de-açúcar e profundidades consideradas, houve maior distribuição na classe de agregados maiores do que $3,35 \mathrm{~mm}$, com destaque para o solo sob mata, seguindo-se o solo sob a aplicação de vinhaça adicionada de torta de filtro; só a aplicação de vinhaça; e, por último, o solo das áreas de cana só com a irrigação por aspersão, na seguinte ordem de valores: 47,0; 37,0; 30,8; e 25,8\%, respectivamente (Figura 1).

O desempenho dos sistemas de manejo com adição de vinhaça e torta e da vinhaça foi creditado à produção de mucilagens, como resultado de maior atividade

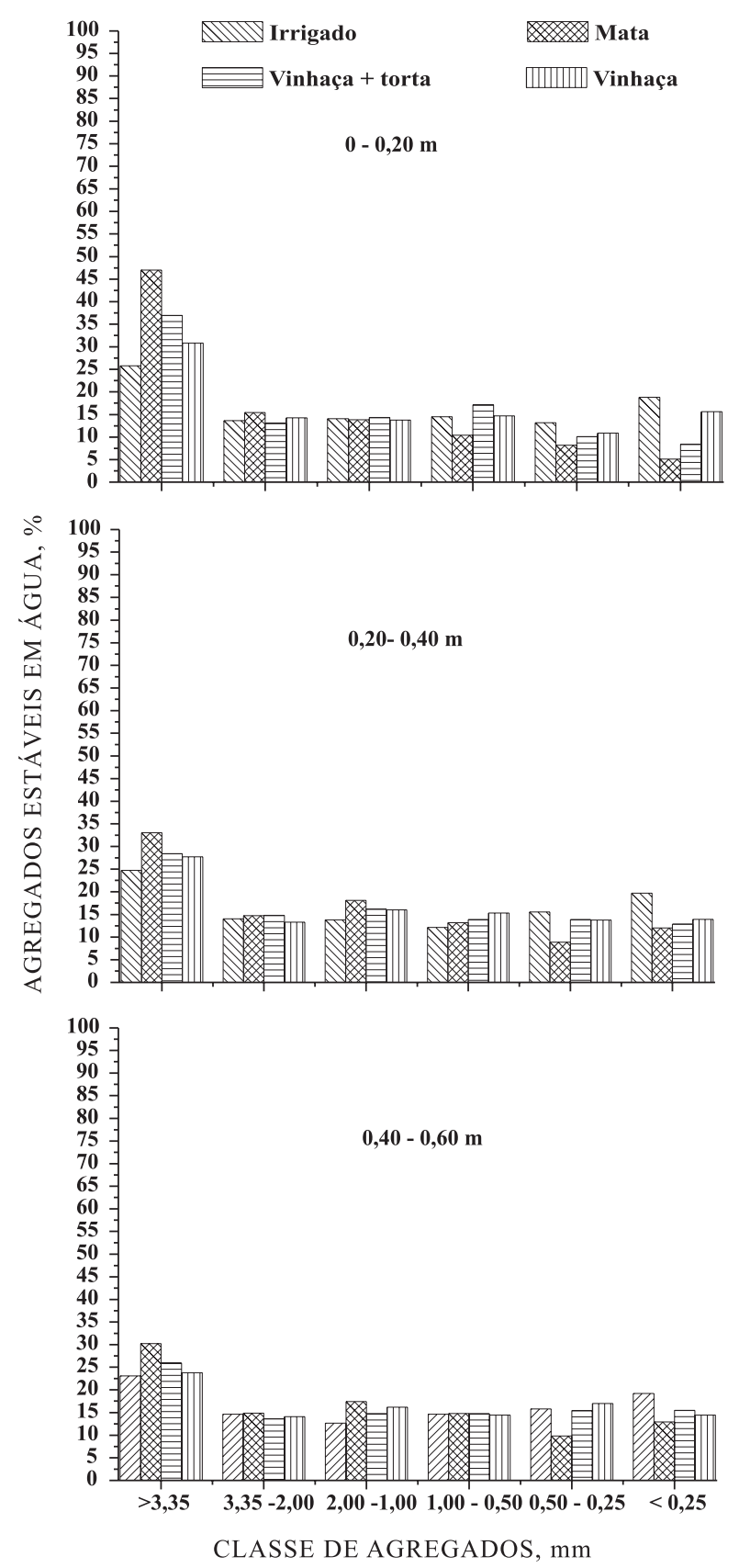

Figura 1. Distribuição dos agregados por peneiramento por via úmida, nos sistemas de manejo e profundidades estudados. 
microbiana em solos que receberam esse resíduo (Camargo et al., 1988). Dessa forma, os sistemas de manejo com cana-de-açúcar que receberam aporte de resíduos orgânicos apresentaram boa estabilidade de agregados em água, principalmente na camada superficial, estando de acordo com alguns autores (Roth et al., 1991; Silva, 2003; Oliveira, 2008).

Pelo que foi discutido, ficou constatado, sobre o peneiramento úmido, que os agregados desse Latossolo cultivado com a cana-de-açúcar resistiram à desintegração do agregado provocada pela explosão deste, ocasionada pela saída do ar (Kemper \& Chepil, 1965). Essa condição dos agregados desenvolvidos sob o cultivo da cana-de-açúcar com adição de resíduos vegetais constitui um indicativo de um bom estado de qualidade desse solo.

\section{Diâmetro médio ponderado e índices de estabilidade dos agregados do solo}

Não houve diferença significativa entre a interação sistemas de manejo e profundidades sobre o DMPs (Quadro 3), indicando que essa propriedade não é adequada para avaliar a estabilidade de agregados de solo sob cultivo em condições de variação de sua umidade.

Já o DMPu mostrou diferenças significativas na comparação das médias para a interação sistemas de manejo e profundidades. Na camada de $0-20 \mathrm{~cm}$, as áreas sob SMVT e, claro, sob mata nativa apresentaram os maiores diâmetros de agregados. Nas camadas de 20-40 e de 40-60 cm, o solo sob mata nativa continuou com os maiores diâmetros de agregado, e os solos sob SMVT e sob SMV igualaram- se, possivelmente devido ao poder da vinhaça de infiltrar-se mais profundamente.

Assim, o DMPu mostrou-se consistente como indicador de qualidade da estrutura do solo com a adição de resíduos orgânicos da cana-de-açúcar.

O IEA e a Agri também se mostraram adequados para a avaliação da estabilidade de agregados. O comportamento geral desses dois índices foi semelhante ao do $\mathrm{DMPu}$ e também indicou melhor estado de agregação do Latossolo estudado, na profundidade de 0-0,20 m, demonstrando uma ação da adição dos resíduos orgânicos da cana-de-açúcar, na forma de aplicação de vinhaça adicionada de torta e da vinhaça isoladamente, sobre a sua camada superficial.

Todas as correlações foram significativas $(p<0,01)$ e positivas, apresentando valores de r de 0,8862; 0,$6304 ; 0,8682 ;$ e 0,9168 , referentes às equações que descrevem as relações entre DMPu vs COT, DMPs vs COT, IEA us COT e Agri vs COT, respectivamente (Figura 2).

Os resultados evidenciam que o processo de estabilização dos agregados desse solo está associado ao teor de matéria orgânica. Na camada superficial, principalmente, à medida que o teor de matéria orgânica diminui, pelo revolvimento excessivo do solo ou pelo baixo aporte de material orgânico proporcionado pelos sistemas de manejo, decresce a estabilidade dos agregados desse solo.

Acredita-se, todavia, que ao efeito da matéria orgânica soma-se a ação dos ciclos sucessivos de umedecimento e secagem. A camada superficial do solo apresenta ciclos de umedecimento e secagem mais

Quadro 3. Diâmetro médio ponderado obtido por peneiração via úmida (DMPu), por peneiração via seca (DMPs), índice de estabilidade de agregados (IEA), percentagem de agregados $>2,0 \mathrm{~mm}$ estáveis em água (Agri), em função dos sistemas de manejo e profundidades estudados

\begin{tabular}{|c|c|c|c|c|c|}
\hline \multirow{2}{*}{ Camada } & \multicolumn{5}{|c|}{ Sistemas de manejo } \\
\hline & SMI & MN & SMVT & SMV & Média \\
\hline $\mathrm{m}$ & \multicolumn{5}{|c|}{ DMP seco $(\mathrm{mm})$} \\
\hline $0-0,20$ & 2,85 & 2,95 & 2,90 & 2,88 & $2,89 \mathrm{a}$ \\
\hline $0,20-0,40$ & 2,82 & 2,93 & 2,88 & 2,86 & $2,87 \mathrm{ab}$ \\
\hline $0,40-0,60$ & 2,81 & 2,91 & 2,87 & 2,84 & $2,86 \mathrm{~b}$ \\
\hline \multirow[t]{2}{*}{ Média } & $2,83 \mathrm{C}$ & $2,93 \mathrm{a}$ & $2,88 \mathrm{~B}$ & $2,86 \mathrm{~B}$ & \\
\hline & \multicolumn{5}{|c|}{ DMP úmido (mm) } \\
\hline $0-0,20$ & $1,62 \mathrm{Da}$ & $2,31 \mathrm{Aa}$ & $1,98 \mathrm{Ba}$ & $1,79 \mathrm{Ca}$ & \\
\hline $0,20-0,40$ & $1,59 \mathrm{Ca}$ & $1,91 \mathrm{Ab}$ & $1,75 \mathrm{Bb}$ & $1,61 \mathrm{Ba}$ & \\
\hline $0,40-0,60$ & $1,54 \mathrm{Ba}$ & $1,83 \mathrm{Ab}$ & $1,64 \mathrm{Bc}$ & $1,61 \mathrm{Bb}$ & \\
\hline & \multicolumn{5}{|c|}{ Índice de Estabilidade de Agregados (\%) } \\
\hline $0-0,20$ & $56,8 \mathrm{Da}$ & $78,3 \mathrm{Aa}$ & $68,3 \mathrm{Ba}$ & $62,1 \mathrm{Cab}$ & \\
\hline $0,20-0,40$ & $56,4 \mathrm{Ca}$ & $65,2 \mathrm{Ab}$ & $60,8 \mathrm{Cb}$ & $59,8 \mathrm{BCab}$ & \\
\hline \multirow[t]{2}{*}{$0,40-0,60$} & $54,8 \mathrm{BCa}$ & $62,9 \mathrm{Ab}$ & $57,1 \mathrm{Bb}$ & $56,7 \mathrm{BCb}$ & \\
\hline & & & AGRI (\%) & & \\
\hline $0-0,20$ & $39,4 \mathrm{Da}$ & $62,5 \mathrm{Aa}$ & $50,0 \mathrm{BA}$ & $45,1 \mathrm{Ca}$ & \\
\hline $0,20-0,40$ & $38,8 \mathrm{Ca}$ & $47,8 \mathrm{Ab}$ & $43,2 \mathrm{BCb}$ & $41,0 \mathrm{BCb}$ & \\
\hline $0,40-0,60$ & $37,7 \mathrm{BCa}$ & $45,1 \mathrm{Ac}$ & $39,6 \mathrm{Bc}$ & $37,9 \mathrm{BCc}$ & \\
\hline
\end{tabular}

Letras maiúsculas iguais na mesma linha e minúsculas iguais na mesma coluna não diferem significativamente pelo teste de Tukey (5\%). Média de quatro repetições. MN: mata nativa; SMI: área irrigada; SMVT: área de vinhaça + torta; SMV: área de vinhaça. 

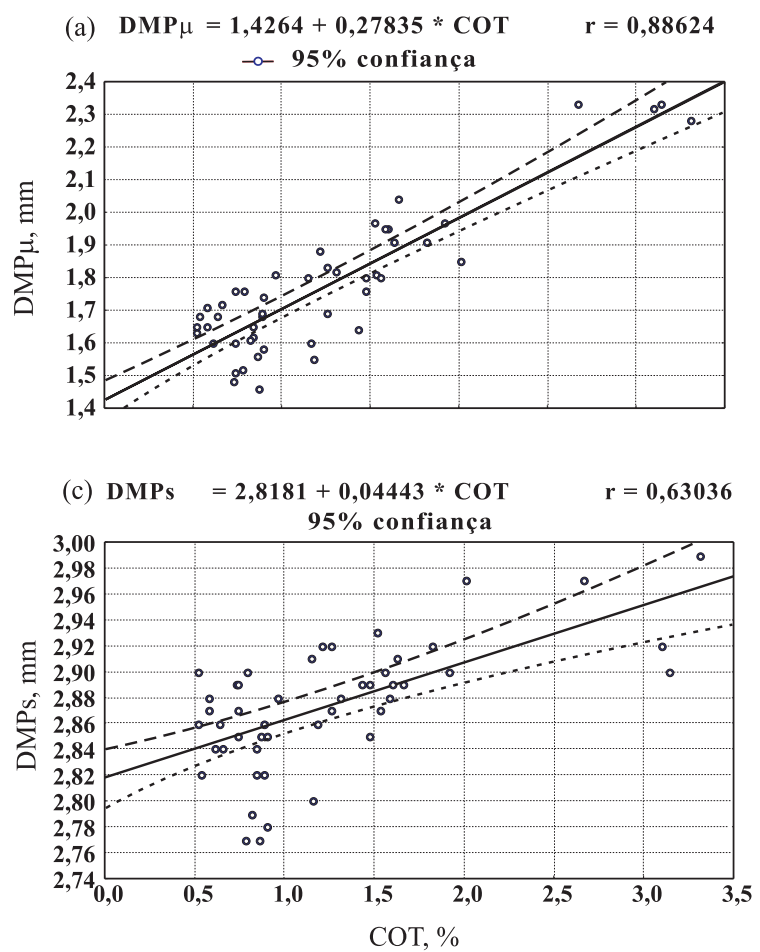
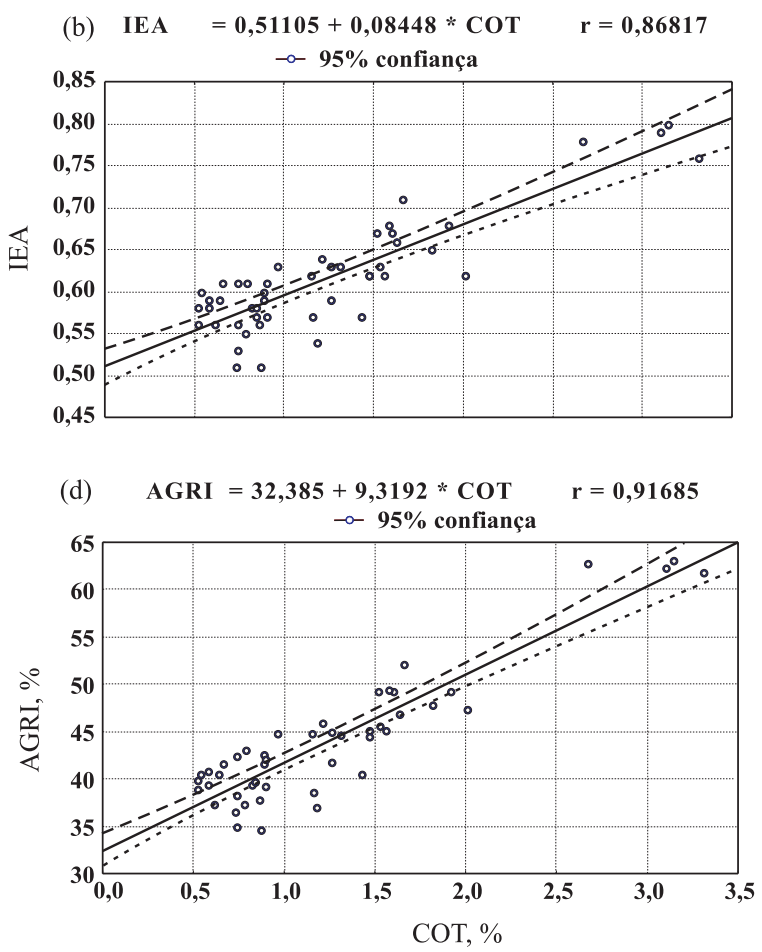

Figura 2. Diâmetro médio ponderado de agregados via úmida (DMP $)$, (a) índice de estabilidade de agregados (IEA), (b) diâmetro médio ponderado de agregados via seca (DMPs), (c) e percentagem de agregados maiores que 2,0 mm estáveis em água (Agri), (d) em função do carbono orgânico total (COT) do solo, para o Latossolo Amarelo distrocoeso.

intensos do que as camadas subsuperficiais. Esses ciclos provocam a desintegração dos ligantes orgânicos e inorgânicos do solo, que resulta na formação de agregados maiores (Horn \& Dexter, 1988; Horn, 1990). Oliveira et al. (1996), investigando a ação dos ciclos de umedecimento e secagem em Latossolos brasileiros, concluíram que seus efeitos sobre a formação e estabilidade de agregados maiores ocorrem nas camadas superficiais. Segundo Hermawan \& Bonke (1997), a variação na estabilidade de agregados e diferentes sistemas de manejo pode ser atribuída a diferenças no teor de C orgânico do solo. Pesquisadores encontraram correlações significativas e positivas entre o teor de C orgânico e os índices de estabilidade de agregados (Roth et al., 1991; Silva et al., 2006; Oliveira, 2008).

\section{CONCLUSÕES}

1. Os sistemas de cultivo da cana-de-açúcar com a aplicação de vinhaça adicionada à torta e da vinhaça isoladamente mostraram-se eficientes no desenvolvimento dos agregados, como constatado pela ocorrência de macroagregados.

2. A adição de resíduos da cana-de-açúcar exerceu ação no aumento do diâmetro médio ponderado dos agregados via úmida.
3. O índice de estabilidade e a percentagem de agregados maiores que 2,0 $\mathrm{mm}$ estáveis em água demonstraram ser propriedades de avaliação da estabilidade de agregados e apresentaram correlações com o teor de $\mathrm{C}$ orgânico total do solo.

4. A estabilização dos agregados na profundidade de 0-0,20 m dependeu do teor de matéria orgânica do solo.

\section{LITERATURA CITADA}

BALESDENT, J.; CHENU, C. \& BALABANE, M. Relationship of soil organic matter dynamics to physical protection and tillage. Soil Tillage Res., 53:215-230, 2000.

CAMARGO, O.A.; VALADARES, J.M.S. \& GERALDI, R.N. Características físicas de solo que recebeu vinhaça. B. Cient. IAC, 14:1-12, 1988 .

CARPENEDO, V. \& MIELNICZUK, J. Estado de agregação e qualidade de agregados de Latossolos Roxos, submetidos a diferentes sistemas de manejo. R. Bras. Ci. Solo, 14: 99$105,1990$.

CHAVES, J.C.D. \& CALEGARI, A. Adubação verde e rotação de culturas. Inf. Agropec., 22:53-60, 2001.

CHINN, C. \& PILLAI, U.P.P. Self-repair of compacted Vertisols from Central Queensland, Australia. Geoderma, 144:491504,2008 
EMPRESA BRASILEIRA DE PESQUISA AGROPECUÁRIA EMBRAPA. Centro Nacional de Pesquisa de Solos. Manual de métodos de análise de solo. 2.ed. Rio de Janeiro, 1997. $212 \mathrm{p}$

EMPRESA BRASILEIRA DE PESQUISA AGROPECUÁRIA EMBRAPA. Centro Nacional de Pesquisa de Solos. Sistema brasileiro de classificação de solos. 2.ed. Rio de Janeiro, 2006. 306p.

GÓES, G.B.; GREGGIO, T.C.; CENTURION, J.F.; AMAURI, N.B. \& ANDRIOLI, I. Efeito do cultivo da cana-de-açúcar na estabilidade de agregados e na condutividade hidráulica do solo. Irriga, 10:116-122, 2005.

GOLCHIN, A.; BALDOCK, J.A. \& OADES, J.M. A model linking organic matter decomposition, chemistry, and aggregate dynamics. In: LAL, R.; KIMBLE, J.M.; FOLLETT, R.F. \& STEWART, B.A., eds. Soil processes and the carbon cycle. Boca Raton, CRC Press, 1997. p.245266. (Advances in Soil Science)

HERMAWAN, B. \& BONKE, A. A. Effect of winter cover crops and successive spring tillage on soil aggregation. Soil Tillage Res., 44:109-120, 1997.

HORN, R. Aggregate characterization as compared to soil bulk properties. Soil Tillage Res., 17:265-289, 1990.

HORN, R. \& DEXTER, R.A. Dynamics of soil aggregation in an irrigated desert loess. Soil Tillage Res., 13:253-266, 1988.

JACOMINE, P.K.T.; CAVALCANTI, A.C.; PESSOA, S.C.P. \& SILVEIRA, C.O. Levantamento exploratório. Reconhecimento de solos do Estado de Alagoas. Recife, Embrapa, Centro de Pesquisas Pedológicas, 1975. 531p. (Boletim Técnico, 35)

KEMPER, W.D. \& CHEPIL, W.S. Size distribution of aggregates. In: BLACK, C.A.; EVANS, D.D.; WHITE, J.L.; ENSMINGER, L.E. \& CLARK, F.E., eds. Methods of soil analysis: Physical and mineralogical properties, including statistics of measurement and sampling. Madison, American Society of Agronomy, 1965. Part 1. p.499-510.

KEMPER, W.D. \& ROSENAU, R.C. Aggregate stability and size distribution. In: KLUTE, A., ed. Methods of soil analysis. Madison, American Society of Agronomy, 1986. Part 1. p.425-442 (Agronomy, 9).

McNABB, D.H.; STARTSEV,A.D. \& NGUYEN, H. Soil wetness and traffic level effects on bulk density and air-filled porosity of compacted boreal forest soils. Soil Sci. Soc. Am. J., 65:1238-1247, 2001.
MILNE, R.M. \& HAYNES,R.J. Comparative effects of annual and permanent dairy pastures on soil physical properties in the Tsitsikamma region of South Africa. Soil Use Manag., 20:81-88, 2004.

NEVES, C.S.V.J.; FELLER, C. \& KOUAKOUA, E. Efeito do manejo do solo e da matéria orgânica em água quente na estabilidade de agregados de um Latossolo Argiloso. Ci. Rural, 36:1410-1415, 2006.

OLIVEIRA, T.S.; COSTA, L.M.; REGAZZI, A.J. \& FIGUEIREDO, M.S. Efeito de ciclos de umedecimento e secagem sobre a estabilidade de agregados em água de quatro Latossolos brasileiros. R. Bras. Ci. Solo, 20:509$515,1996$.

OLIVEIRA, V.S. Compactação em solos coesos dos tabuleiros costeiros de Alagoas. Recife, Universidade Federal Rural de Pernambuco, 2008. 81p. (Tese de Doutorado)

RICHARD, G.; COUSIN, I.; SILLON, J.F.; BRUAND, A. \& GUÉRIF, J. Effect of compaction on the porosity of a silty loam: Influence on unsaturated hydraulic properties. Eur. J. Soil Sci., 52:49-58, 2001.

ROSCOE, R. \& MACHADO, P.L.O.A. Fracionamento físico do solo em estudo da matéria orgânica. Rio de Janeiro, Embrapa Solos, 2002. 86p.

ROTH, C.H.; CASTRO FILHO, C. \& MEDEIROS, G.B. Análise de fatores físicos e químicos relacionados com agregados de um Latossolo Roxo distrófico. R. Bras. Ci. Solo, 15:241$248,1991$.

SAEG. Sistema para análises estatísticas. Versão 9.0. Viçosa, MG, Fundação Arthur Bernardes, 2005.

SILVA, A.J.N. Alterações físicas e químicas de um Argissolo Amarelo Coeso sob diferentes sistemas de manejo com cana-de-açúcar. Porto Alegre, Universidade Federal do Rio Grande do Sul, 2003. 120p. (Tese de Doutorado)

SILVA, A.J.N.; CABEDA, M.S.V. \& CARVALHO, F.G. Matéria orgânica e propriedades físicas de um Argissolo Amarelo Coeso sob sistemas de manejo com cana-de-açúcar. R. Bras. Eng. Agric. Amb., 10:579-585, 2006.

STATSOFT, Statistica: Data analysis software system. Version 6, 2001.

WENDLING, B.; JUCKSCH, I.; MENDONÇA, E.S. \& NEVES, J.C.L. Carbono orgânico e estabilidade de agregados de um Latossolo Vermelho sob diferentes manejos. Pesq. Agropec. Bras., 40:487-494, 2005.

WOHLENBERG, E.V.; REICHERT, J.M.; REINERT, D.J. \& BLUME, E. Dinâmica da agregação de um solo francoarenoso em cinco sistemas de culturas em rotação e em sucessão. R. Bras. Ci. Solo, 28:891-900, 2004. 